\title{
Corela
}

Cognition, représentation, langage

HS-34 | 2021

Les noms sous-spécifiés en français: du lexique au discours

\section{Le souci est, c'est que cette modification se fait à l'insu de l'utilisateur}

A propos d'une réalisation non prototypique des constructions spécificationnelles du français

\section{Frédéric Sabio}

\section{CpenEdition}

\section{Journals}

Édition électronique

URL : https://journals.openedition.org/corela/13812

DOI : 10.4000/corela.13812

ISSN : $1638-573 X$

Éditeur

Cercle linguistique du Centre et de l'Ouest - CerLICO

Référence électronique

Frédéric Sabio, «Le souci est, c'est que cette modification se fait à l'insu de l'utilisateur », Corela [En ligne], HS-34 | 2021, mis en ligne le 09 décembre 2021, consulté le 13 décembre 2021. URL : http:// journals.openedition.org/corela/13812 ; DOI : https://doi.org/10.4000/corela.13812

Ce document a été généré automatiquement le 13 décembre 2021.

Corela - cognition, représentation, langage est mis à disposition selon les termes de la licence Creative Commons Attribution - Pas d'Utilisation Commerciale - Partage dans les Mêmes Conditions 4.0 International. 


\section{Le souci est, c'est que cette modification se fait à l'insu de l'utilisateur}

A propos d'une réalisation non prototypique des constructions spécificationnelles du français

\section{Frédéric Sabio}

\section{Introduction}

1 Nous proposons de rendre compte de certains énoncés non canoniques du type [ $\mathrm{N}$ est c'est X], tels que :

[1] on dit que c'est a cause de nos idées néolibérales, productivistes, mondialistes, immigrationistes que tout va mal... le fait est c'est qu'on est un système ultracollectivistes, centralisé, antilibéral à tout les étages [web ${ }^{1}$ ]

[2] Et la preuve en est c'est que Diaz est bien plus fort aujourd'hui qu'il y a 3 mois. Donc les critiques constructives ont du bon. [web ${ }^{2}$ ]

2 Nous mentionnerons également les énoncés dans lesquels la première occurrence $d u$ verbe être est au participe présent, [N étant c'est $\mathrm{X}]$ :

[3] Alors plus sérieusement, le film est globalement bon. Le titre nous promet une bataille épique et bataille épique il y a. Le soucis étant c'est que l'ensemble souffre de grosses coupures au montage nuisant à la cohérence de la narration. [web ${ }^{3}$ ]

3 Ces structures sont extrêmement intrigantes d'un point de vue syntaxique : l'élément $\mathrm{X}$ (qui, comme on le verra plus bas, est le plus souvent une Que-C.) est précédé de 2 formes du verbe être (par ex. est + c'est) là où on en attendrait une seule dans les constructions spécificationnelles à nom sous-spécifiét du français normatif ${ }^{5}$.

L'objectif de cet article sera double :

5 Dans une première partie, nous nous interrogerons sur l'attestation de ces tournures en français moderne, en examinant en particulier le type de noms sous-spécifiés (Legallois $2006,2008)$ qui s'y rencontrent le plus fréquemment. 
6 Dans la suite, nous nous demanderons quel statut grammatical on peut donner à ces énoncés. L'examen portera d'abord sur les constructions spécificationnelles du français standard (ex. le problème est qu'il est parti / le problème c'est qu'il est parti), pour aborder ensuite le cas particulier des réalisations non canoniques en est c'est (le problème est c'est qu'il est parti).

\section{A- données étudiées et occurrences relevées}

7 Le corpus réuni pour cette étude totalise un ensemble de 241 énoncés en $N$ est c'est $X, N$ était c'est $X$ et $N$ étant c'est $X$. Voici quelques indications sur la manière dont nous avons constitué notre corpus d'étude.

\section{1-Formes non retenues}

8 - Afin de pouvoir raisonner sur des formes suffisamment homogènes, nous avons choisi de ne pas retenir pour cette étude les constructions spécificationnelles dont la partie lexicale initiale est constituée d'un adjectif nominalisé (le pire, l'intéressant...), ou d'une séquence $\mathrm{N}$ + Adj ou Adj. + N (la chose importante, le premier problème...).

9 - Un point crucial était de pouvoir distinguer les structures en est c'est d'un autre type syntaxique, exclu de cette étude, qu'on peut illustrer par :

[4] Le problème est, c'est une 15-vitesse [web]

[5] Ma question est c'est quoi une tempête ? [web]

10 Dans ces exemples, la séquence introduite par c'est constitue une construction syntaxique formellement autonome (c'est une 15-vitesse, c'est quoi une tempête?) dans laquelle l'élément c'est alternerait sans difficulté avec un autre élément verbal ( $L e$ problème est, il s'agit d'une 5-vitesse; Ma question est qu'appelle-ton une tempête ?). Les deux séquences successives de ces énoncés entretiennent une relation purement parataxique, présentant ce que C. Blanche-Benveniste (2010) a désigné, à la suite de B. Martinie \& F. Sitri (1999), un « effet deux points ", que l'on pourrait de fait représenter avec la ponctuation suivante :

[6] Le problème est : c'est une 15-vitesse

Ma question est : c'est quoi une tempête?

11 Dans ces structures, le fait principal à retenir est que les deux formes successives de être sont indispensables à l'acceptabilité des énoncés :

[7] Le problème est c'est une 5 vitesse - *Le problème est une 5 vitesses

Ma question est c'est quoi une tempête ? - *Ma question est quoi une tempête?

12 A l'inverse, dans les structures en est c'est qui seront étudiées ici, le verbe être donne l'impression d'avoir été "indûment " dupliqué, si bien que le fait de n'en conserver qu'une occurrence ne poserait aucun problème et serait pour le coup parfaitement normatif. Ainsi, à partir de [1] on pourrait avoir :

[8] le fait est qu'on est un système ultra-collectiviste

13 - Enfin, nous avons écarté les séquences en c'est c'est, qui sont d'une part extrêmement minoritaires, et qui peuvent par ailleurs être conçues comme des phénomènes de " piétinement » graphique assimilables à des disfluences :

[9] mais le souci c'est c'est que le passage se fait difficilement et une fois sur deux.

$\left[\mathrm{web}^{6}\right.$ ] 


\section{2-Corpus étudiés et occurrences relevées}

$$
\begin{aligned}
& \text { totalisant environ 4.000.000 mots de françals } \\
& \text { - Frantext intégral (251.000.000 mots écrits). }
\end{aligned}
$$

17 - Le French Web Corpus ${ }^{7}$ (FWC). Ce corpus écrit, qui compte pratiquement 10 milliards de mots, a été constitué en avril 2012 (Jakubíček et al. 2013). Il a été interrogé au moyen de l'outil de recherche Sketch Engine ${ }^{8}$.

18 Afin d'orienter notre recherche, nous nous sommes fondé sur les inventaires de noms sous-spécifiés disponibles dans la littérature ${ }^{9}$ : celui dressé par Blanche-Benveniste $(1992)^{10}$, et celui, bien plus exhaustif, de Legallois (2008). Nous avons en particulier veillé à nous concentrer sur les noms que Legallois \& Gréa $(2006,170)$ présentent comme les plus fréquemment associés aux constructions à nom sous-spécifié (désormais « Nss ») en « être + Que-C. » ou en « être + de Inf ». condition conseil conséquence conviction crainte danger défaut différence difficulté effet enjeu ennui fait hantise hic idéal idée impression inconvénient inquiétude intention intérêt logique moyen mystère objectif objection opinion paradoxe particularité peur preuve principe priorité problème projet question raison règle regret reproche résultat risque sentiment solution souci stratégie suggestion surprise vérité

21 Résultats : le corpus oral et la base écrite Frantext n'ont fourni aucune occurrence de la structure recherchée ${ }^{11}$. Le FWC en a livré 63 exemples.

22 Après avoir vérifié à partir du FWC que les données issues du web constituaient une bonne source d'occurrences, nous avons procédé à un relevé informel à partir du moteur de recherche Google. Il est important de préciser qu'en raison du caractère non stabilisé de cette base de données, notre étude se condamnera à demeurer essentiellement qualitative : même si nous donnerons quelques indications globales sur la fréquence relative des attestations en fonction des différents lexèmes, il ne sera pas possible d'établir des comptages précis ${ }^{12}$.

23 Cette recherche informelle sur le web a livré 178 occurrences supplémentaires, provenant essentiellement de blogs et fils de discussion, ce qui nous conduit à un corpus total de 241 formes.

\section{3-Remarques préliminaires sur les données recueillies}

Avant d'aller plus loin dans la description des énoncés, nous souhaitons faire deux remarques sur le statut que l'on doit attribuer à ces données :

\section{a- Faits de compétence vs. accident de performance}

Comme l'indique la section précédente, les énoncés en est c'est apparaissent très marginaux quant à leur fréquence : 241 exemples sur plusieurs milliards de mots, c'est bien peu. Cette rareté nous oblige à prendre position sur le fait qu'on se trouve bien en 
présence d'authentiques structures linguistiques plutôt que de simples faits de disfluence scripturale, dont on peut penser qu'ils doivent être relativement nombreux dans les écrits informels du web ${ }^{13}$. Il n'y a pas de réponse évidente à cette question ; je me fonderai sur les quatre faits suivants pour faire l'hypothèse qu'on a bien affaire à une structure conventionnalisée, et non à des suites langagières accidentelles analysables comme faits de performance ${ }^{14}$ :

a- Comme on le verra plus loin, la structure est surtout attestée avec un petit nombre de lexèmes sous-spécifiés tels que preuve, fait, problème et souci: cette relation privilégiée entre un schème d'énoncé et certaines unités lexicales peut être interprétée comme une forme de conventionnalisation, qui rend du coup moins plausible l'hypothèse de l'accident de performance.

b-Si la plupart des énoncés recueillis paraissent clairement non prototypiques, il existe au moins un lexème qui, lui, paraît attesté de longue date, même dans des écrits de tonalité cérémonieuse : il s'agit du nom preuve, qui fournit de très nombreux exemples en la preuve en est c'est que ou la preuve étant c'est que. Par exemple, le passage suivant, qui date du début du $19^{\mathrm{e}}$ siècle, en présente trois occurrences successives :

[10] Oui, mon divin sauveur, c'est par ce motief que je déteste tous mes péchés, la moindre même de mes infidélités; la preuve en est, c'est que quand je pourrois les cacher pour jamais à votre lumière éternelle, je ne voudrois pas les commettre ; la preuve en est, c'est que quand il n'y auroit point d'enfer, je ne voudrois pas les éviter avec moins de soin : la preuve en est, c'est que quand, par une supposition impossible, cela devroit m'ouvrir le ciel, je ne voudrois point du ciel même à ce prix, et par ce moyen ; [1805, Traité abrégé de la sainte volonté de Dieu..., par un religieux de la Val-Sainte de Notre Dame de la Trappe, p.185]

En se fondant sur l'existence de tels exemples, on peut faire l'hypothèse que les énoncés les plus atypiques de notre corpus ne font qu'exploiter avec un lexique différent un type de structure qui, dans le cas de preuve, parait relativement bien « installé » dans la syntaxe du français.

c- Les arguments de nature intonative paraissent également jouer en la faveur de la reconnaissance de ces énoncés en tant que structures : lorsqu'on demande à des locuteurs d'en réaliser la lecture à voix haute ${ }^{15}$, la prononciation du premier est se révèle plus fortement marquée que celle du c'est subséquent: elle est accentuée, réalisée par un contour intonatif majeur et, dans certains cas, suivie d'une pause : les deux occurrences de être semblent donc relever de deux unités intonatives différentes :

[11] Le fait est / c'est qu'il y a des chemins de traverses qui ont été pris $\backslash\left[\mathrm{web}^{16}\right]$

Ceci revient à dire que les locuteurs ne prononcent pas les deux formes de être comme une suite d'éléments simplement répétés, dans une forme de "piétinement» paradigmatique (comme ce serait le cas pour je je je), mais qu'ils tendent à les associer à deux entités structurelles distinctes. Dans le cadre méthodologique de l'approche pronominale étendue à la macrosyntaxe (Debaisieux dir. 2013), on dirait que le premier est se trouve placé à la fin de l'élément Prénoyau de l'énoncé, alors que la forme c'est introduit la séquence Noyau :

$$
\text { [12] [Le fait est }]_{\text {Prénoyau }}[\text { 'est qu'il y a des chemins de traverses qui ont été pris }]_{\text {Noyau }}
$$

d- Le dernier argument, qui est en quelque sorte indirect, concerne l'existence en anglais de constructions qui semblent assez proches des structures françaises décrites ici : il s'agit de tournures diversement désignées comme "reduplicative copulas", "double is », «double copula " ou "is is» constructions. Bien attestées depuis la 
seconde moitié du 20e siècle en anglais américain, australien et néo-zélandais, ces tournures tendent à se généraliser à d'autres variétés, comme l'anglais britannique (Andersen 2002). Depuis la première mention de ces structures par D. Bolinger en 1987, de nombreuses études en ont été proposées ${ }^{17}$, dont la plupart soulignent que les énoncés en question ne sont en aucun cas assimilables à des disfluences sur le plan prosodique [voir notamment Coppock et al. 2006].

Comme dans les structures françaises correspondantes, les constructions anglaises en is is présentent la double caractéristique d'introduire un lexème sous-spécifié de type thing, problem, issue, et de dupliquer le verbe copule (is). On peut à cet égard comparer :

[13] The funny thing
Lit:Lachose amusante est est que Lisa était là aussi
La chose

33 Comme en français (voir plus loin), les constructions à double copule de l'anglais sont surtout attestées devant une that-clause, mais se rencontrent aussi devant une séquence en to + infinitif, ou un $\mathrm{SN} .^{18}$

\section{b- Données écrites vs. données orales}

34 - Le fait que les occurrences relevées soient présentes dans des corpus écrits (FWC + Google) n'implique évidemment pas pour nous qu'on ait affaire à des tournures typiques de l'écrit ; c'est plutôt la quantité considérable des données du web, conjuguée à la relative marginalité de la tournure, qui fait qu'on aura statistiquement plus de chance d'en trouver des occurrences dans le web que dans les corpus oraux, de dimension infiniment plus modeste.

35 - Quant à l'absence de la tournure dans la base Frantext, qui comprend des textes écrits formellement plus académiques, elle doit s'expliquer essentiellement par le caractère très informel / non normatif de la tournure en question, et sans doute aussi par le fait que les échanges explicatifs / argumentatifs font des forums de discussion et autres blogs internet une source particulièrement propice à l'emploi de ces structures.

\section{B- Quelques tendances lexicales et grammaticales}

Voici quelques caractéristiques générales des énoncés recueillis, qui touchent à la répartition lexicale, aux catégories grammaticales, aux temps verbaux et à la ponctuation.

\section{1- Type de lexème sous-spécifié}

\section{a- Dans le French Web Corpus (63 occ.)}

37 Nous mentionnons en premier lieu le corpus FWC qui, en tant que corpus fermé, permet d'évaluer précisément la fréquence relative des lexèmes employés : 11 noms sous-spécifiés apparaissent, avec une très forte domination du nom preuve : 


\begin{tabular}{|c|c|}
\hline LEXÈME & OCCURRENCES \\
\hline preuve & $44(=70 \%$ de l'ensemble $)$ \\
\hline problème & 6 \\
\hline chose & 4 \\
\hline fait & 2 \\
\hline $\begin{array}{c}\text { avis différence hic inconvénient } \\
\text { objectif solution souci }\end{array}$ & 1 \\
\hline
\end{tabular}

\section{b- Dans Google (178 occ.)}

En ce qui concerne la recherche menée à partir de Google, il serait peu pertinent de fournir des indications chiffrées trop précises, en raison du caractère non stabilisé de la base. Nous nous contenterons de proposer une répartition tripartite approximative dans le tableau ci-dessous : ++ indique les lexèmes les mieux attestés, dont nous avons relevé plus de 30 exemples ; + indique les lexèmes moins fréquents mais qui demeurent relativement bien attestés (plus de 10 ex.); les lexèmes donnés sur la dernière ligne sont représentés marginalement (2-4 exemples).

\begin{tabular}{|l|c|}
\hline \multicolumn{1}{|c|}{ LEXÈME } & OCCURRENCES \\
\hline fait preuve problème souci & ++ \\
\hline chose raison & + \\
\hline $\begin{array}{l}\text { avantage but défaut différence difficulté hic idéal } \\
\text { idée paradoxe question risque solution regret }\end{array}$ \\
\hline
\end{tabular}

Sur l'ensemble des données, nous relevons donc que :

a- Les 4 lexèmes les mieux représentés sont : preuve, fait, problème, souci (tous présents à la fois dans le FWC et dans Google) :

Le nom preuve est de loin le mieux attesté dans les énoncés en est c'est. La forme avec déterminant (la preuve en est; cf. [2] et [10]) semble plus fréquente que celle sans déterminant, qui est toutefois bien attestée aussi. Par ex. :

[14] si tu la connaissais un peu mieux, tu verrais qu'elle n'est pas bête du tout et qu'elle a beaucoup de talent, preuve en est c'est qu'elle est toujours là et qu'elle a su se faire apprécier par un public de plus en plus vaste. [...] [FWC]

La version sans pronom (preuve est, la preuve est) est en revanche très marginale, du moins lorsque être est conjugué au présent [voir plus loin] :

[15] nous étions mieux préparé que eux et la preuve est c'est que nous marquons les deux buts vent contre [ web $\left.^{19}\right]$

Les noms fait ([1] et [16] ci-dessous), souci ([3] et [17] ci-dessous $\left.{ }^{20}\right)$ et problème ([18] et [19] ci-dessous) sont largement attestés aussi :

[16] J'ai croisé des «connards » en agriculture, des têtus, des bornés etc , tout ce que vous voudrez comme dénominateur négatifs. mais le fait est c'est que ce sont des agriculteurs qui nourrissent les gens même s'ils le font mal ${ }^{21}$

[17] J'ai commencer le 01/04/08 a travailler au sein d'une petite entreprise de bâtiment et j'arrive bientôt à la fin de ma période d'essai (31/05/08) mais le soucis est, c'est que je n'es toujours pas eu ma paye du mois d'avril et on arrive fin du mois de mai avec en plus cette paye ci. Que dois-je faire? [web ${ }^{22}$ ] 
[18] les ventes d'imprimés diminuent, au grand malheur des éditeurs qui faisaient leur marge dessus, le problème est c'est qu'en parallèle, les consommateurs souhaitent que le prix de vente des ebooks soit bas. [FWC]

[19] il y a trois jours $\mathrm{j}$ ai fais changé mes disques de frein et plaquettes de frein avant et arriere, hier soir les voyant frein et stop se son allumé donc je me suis arrêté, et en regardant se matin $\mathrm{j}$ ai vu que sa fuyait au niveau de la roue droite arriere, et il $\mathrm{n}$ y a plus de liquide de frein dans le réservoir, mes freins ont été fais chez norauto, mon probleme est c est que je vis a $35 \mathrm{~km}$ du norauto qui a changé mes freins, comment faire? [web ${ }^{23}$ ]

b- Les noms chose ${ }^{24}$ et raison sont moins fréquents mais assez bien attestés :

[20]- je craignais sa réponse. Je craignais qu'elle me dise que je l'avais déjà perdu. Je ne le supporterais pas.. " La chose est c'est que notre amitié ne sera jamais plus pareille! Elle ne l'est déjà plus... [FWC]

[21] Mon village a pour emblème l'écureuil, la raison est c'est qu'il y en a partout aux alentours. Il y a des roux et des marrons clairs. Mais je n'ai réussi qu'une seule fois à prendre une photo, comme tu dis ils sont rapides. [web ${ }^{25}$ ]

[22] Le droit patrimonial est un droit de propriété qui a la particularité d'être temporaire. La raison en est c'est que le droit de la propriété intellectuelle vise à favoriser la création en accordant une prérogative extraordinaire pour les auteurs, à savoir un monopole. [web ${ }^{26}$ ]

c- Nous relevons 16 lexèmes qui ne livrent pas plus de 4 ou 5 occurrences chacun : avantage, avis, but, défaut, différence, difficulté, hic, idéal, idée, inconvénient, objectif, paradoxe, question, risque, solution, regret. Par exemple :

[23] la recette du pain d'épice est rapide à réaliser et l'avantage est c'est qu'il se garde plusieurs jours [web ${ }^{27}$ ]

[24] Donc l'idée est c'est vraiment de te permettre d'accéder à ces lieux magiques qui te font rêver. [web ${ }^{28}$ ]

[25] Le défaut est, c'est que le gouvernement japonais n'a pas fait son travail de sécurisation du site afin de protéger son peuple. [web ${ }^{29}$ ]

[26] Donc ma question est c'est a qui appartient cette tombe? (j'ai pensé a Big Boss ou

Gray Fox, mais je suis sur de rien...) [web $\left.{ }^{30}\right]$

Enfin, 40 lexèmes de notre inventaire de départ (qui en comprenait 61) ne sont pas du tout représentés.

PB : chercher la construction en spécifiant le lexème (cf. liste plus haut : assez modeste) contraint trop : or la structure est tellement spéciale qu'il fallait la projeter telle quelle sur le corpus

On voit donc qu'un tout petit nombre de lexèmes a des affinités avec la tournure en est c'est. Mais il est évidemment impossible d'en donner une liste close, dans la mesure où d'assez nombreux lexèmes paraissent susceptibles de se retrouver dans cet emploi, ne serait-ce que de manière très occasionnelle.

\section{2- Catégorie du groupe placé après est c'est}

Tous lexèmes confondus, on note la prééminence des Que-Constructions (plus de 93\% du total), et la marginalité des SN : 


\begin{tabular}{|l|l|l|}
\hline $\begin{array}{c}\text { Catégorie apparaissant } \\
\text { après est c'est }\end{array}$ & \multicolumn{1}{|c|}{ occs } & \multicolumn{1}{c|}{ Lexèmes attestés } \\
\hline Que-C. & $225(93 \%)$ & [lexèmes variés] \\
\hline de INF & 11 & but chose idéal idée problème regret \\
\hline Construction verbale & 3 & problème question solution \\
\hline $\mathrm{SN}$ & 2 & problème raison \\
\hline
\end{tabular}

50 A titre d'illustration, voici 4 énoncés présentant le nom problème dans chacune des catégories :

[27] Le gros problème, est c'est [qu'il y a des enfants de 8-10 ans par milliers qui utilisent ce jeu, alors que théoriquement ils n'ont pas le droit. $]_{\text {Que-c }}\left[\right.$ web $\left.^{31}\right]$

[28] Notre problème est c'est [d'apprendre à gérer notre intelligence. $]_{\text {de INF }}\left[\mathrm{web}^{32}\right]$

[29] Mon problème est c'est [quand je jou en solo le son est avnt l'image ! $]_{\mathrm{CV}}\left[\mathrm{web}^{33}\right.$ ]

[30] Mon problème est c'est [les soirées entre copains $]_{S N}$, depuis mon adolescence j'ai des soucis avec l'alcool festif, je me retrouve en soirée et ça dérape [web $\left.{ }^{34}\right]$

\section{3- Temps de être}

51 La forme c'est est invariablement au présent ${ }^{35}$. La forme de être qui précède est en revanche susceptible d'une certaine variation morphologique : est c'est, étant c'est, était c'est.

\section{a- Est / étant}

Si on s'en tient aux 4 lexèmes les mieux représenté dans notre corpus (preuve, problème, fait, souci), on note que :

53 - Pour le nom preuve : la preuve étant c'est que est assez bien représenté, à l'inverse de la version avec pronom clitique, la preuve en étant c'est que, qui n'est pas présente du tout. On a donc une tendance distributionnelle assez nette quant à la présence du clitique par rapport au temps verbal : avec le présent, présence du pronom (la preuve en est c'est que); avec le participe présent, absence de pronom (la preuve étant c'est que).

54 - Le nom problème avec participe présent est bien attesté :

[31] J'ai un problème avec un employeur, j'etais en cdd jusqu'a fin octobre, et depuis ce jour la, j'attend toujours mon solde de tout compte, le probleme etant c'est que je ne peux pas me deplacer au bureau de mon ancien patron car je suis a l'etranger pour l'instant. [web ${ }^{36}$ ]

55 - Le nom souci est également bien attesté avec un participe présent, et même davantage qu'avec le présent. Cf. [3] et l'ex. suivant :

[32] Aujourd'hui, j'ai voulu manœuvrer pour me garer correctement sur une place de parking. Le souci étant c'est qu'en fin de marche arrière, j'ai tapé dans un véhicule. [web ${ }^{37}$ ]

56 - A la différence des lexèmes précédents, fait est en revanche absent sous la forme le fait étant c'est que, ce qu'on peut mettre en relation avec le caractère très fortement figé de le fait est que (cf. Lenepveu 2009).

\section{b- Est / était}

Les formes en N était c'est que sont marginales; quelques occurrences tout de même :

[33] c'est vrai que de toute façon entre eux ça pouvait que tourner au désastre la preuve en était c'est qu'elle se retrouvait aux urgences [FWC] 
[34] le seul Hic était c'est qu'on avait une salle de bain privative mais au cours des 4 nuits/5jours le service de nettoyage n'était pas passé dans la chambre [web ${ }^{38}$ ]

\section{4- Ponctuation} afin de voir si une tendance systématique à la démarcation graphique pouvait être dégagée. Nous nous sommes limité aux lexèmes preuve, problème, fait et souci. Il apparait que les scripteurs n'utilisent aucun signe de démarcation graphique dans $61 \%$ des cas. La démarcation est à l'inverse faite dans $39 \%$ des cas, quasi-exclusivement avec la virgule. Au-delà de cette tendance générale, les différents lexèmes ont des comportements spécifiques : les noms fait, problème et souci se conforment à la tendance générale (l'absence de signe de ponctuation est majoritaire). En revanche, preuve provoque majoritairement (dans $63 \%$ des cas) l'usage d'une virgule.

Au-delà de ces considérations générales, nous souhaiterions revenir de manière précise sur le statut syntaxique de ces constructions non prototypiques ${ }^{39}$.

\section{C- ELEMENTS DE DESCRIPTION SYNTAXIQUE}

nous semblait difficile d'aborder la syntaxe des constructions non canoniques en est c'est sans rappeler au préalable certaines difficultés que posent les formes spécificationnelles usuelles, sans duplication du verbe être (le problème est que, le problème c'est que...). En effet, pour « prototypiques » que soient les exemples de ce type, leur description grammaticale est très loin d'aller de soi. En particulier, l'élément verbal (est, c'est) devra être regardé de près, et son caractère " copulatif » précisé. Ce n'est qu'après avoir mentionné certaines particularités de ces structures qu'on pourra reprendre la description formelle des énoncés en est c'est.

\section{1- Les constructions spécificationnelles prototypiques}

Dans la littérature linguistique, les noms sous-spécifiés sont généralement appréhendés sur une base davantage sémantique, informationnelle ou textuelle que strictement syntaxique. Telle est par exemple l'orientation de Schmid $(2000,4)$, qui les définit comme "abstract nouns that have [...] the potential for being used as conceptual shells for complex, proposition-like pieces of information". Il semble n'y avoir aucun véritable consensus sur l'analyse grammaticale qu'on doit réserver à ces énoncés. Deux pistes descriptives distinctes peuvent être envisagées :

1) celle qui s'inscrit, de manière assez classique, dans la «syntaxe des fonctions ", et qui fait de être un verbe en emploi copulatif qui est grammaticalement relié à un Sujet et un Objet / Attribut.

2) une piste, davantage constructionnelle, qui propose d'établir un lien entre les constructions à Nss et les énoncés pseudo-clivés prototypiques (type : ce que j'aime c'est qu'il est toujours souriant). 


\section{a- Constructions spécificationnelles et fonctions syntaxiques}

64 La première piste de description grammaticale amène à se demander dans quelle mesure la syntaxe de ces constructions peut être adéquatement décrite à partir du verbe être. Pour répondre affirmativement à cette question, il faudrait pouvoir montrer que les deux séquences entourant le verbe dépendent syntaxiquement de celui-ci, et tenter de préciser la fonction qu'elles exercent.

Il apparait que, pour bon nombre d'observateurs, les énoncés spécificationnels à Nss peuvent être ramenés à des séquences Sujet-Verbe-Objet ${ }^{40}$ : par exemple, dans leurs travaux sur l'anglais, Brenier \& Michaelis $(2005,48)$ considèrent - mais sans en donner de justification précise - que la séquence initiale en the thing ou the problem est un SN Sujet, alors que la séquence placée à droite de la copule is exerce une fonction de complément.

Pour le français, l'idée que le nom sous-spécifié puisse constituer le Sujet de être est proposée par Lenepveu (2009) à propos des séquences en le fait est que : celle-ci souligne que le lexème fait «appartient à cette classe de noms qui ont la (...) propriété de pouvoir fonctionner aussi comme sujet grammatical du verbe être » (211). De même, pour Rouveret $(1998,23)$, «les phrases spécificationnelles ont pour sujet une expression nominale définie, fonctionnant comme l'étiquette d'une liste, et pour attribut un terme qui renvoie à un élément de cette liste ».

En rupture avec ces conceptions, plusieurs auteurs (Moreau 1976, Van Peteghem 1991, Blanche-Benveniste 1992) ont souligné que cette analyse comportait plusieurs difficultés.

\section{1- le SN initial est-il bien le Sujet?}

La description du SN sous-spécifié initial comme Sujet de être se heurte à un phénomène de décalage plusieurs fois mentionné (cf. Moreau 1976, Blanche-Benveniste 1992) entre propriétés morphologiques, topologiques et syntaxiques :

69 - La topologie et la morphologie plaident en faveur de l'analyse comme Sujet, en raison de l'emplacement linéaire et des phénomènes d'accord généralement observés ${ }^{41}$. C'est ainsi que dans les exemples suivants, les $\mathrm{SN}$ les soucis et la contradiction et le problème pourraient, en tant que donneurs d'accord pluriel antéposés au verbe, être vus comme Sujets de être.

[35] Les soucis sont que ce produit n'est pas distribué en France et que le vaccin ne protège pas contre toutes les souches de la maladie (...) [web]

[36] La contradiction et le problème sont donc que l'individu moderne veut être défendu dans tous les domaines (...) [web]

En revanche, certaines propriétés syntaxiques, qui touchent aux possibilités de pronominalisation et de clivage, viennent fragiliser cette analyse :

-Pronominalisation:

72 Le SN initial n'accepte pas d'être pronominalisé dans le paradigme spécifique du Sujet (il, elle,...) (Blanche-Benveniste 1992, 7)

[37] Le problème est qu'il est parti avant midi

*Il est qu'il est parti avant midi

[38] La preuve (en) est qu'il est parti avant midi

*Elle (en) est qu'il est parti avant midi 
Les proformes interrogatives en qu'est-ce qui sont impossibles aussi :

[39] *Qu'est-ce qui est qu'il est parti avant midi ?

Remarquons que l'on peut opposer ces exemples à d'autres emplois (à interprétation attributive et non spécificationnelle) pour lesquels la pronominalisation serait tout à fait recevable :

[40] Ton problème est difficile à résoudre / il est difficile à résoudre / qu'est-ce qui est difficile à résoudre?

[41] La meilleure preuve a été apporté hier / elle a été apportée hier / qu'est-ce qui a été apporté hier?

\section{-Clivage:}

Les Sujets français peuvent régulièrement être clivés sous la forme c'est... qui, ce qui est impossible pour les SN initiaux de nos exemples :

[42] Le problème est qu'il est parti avant midi

${ }^{*}$ C'est le problème qui est qu'il est parti avant midi

[43] La preuve (en) est qu'il est parti avant midi

${ }^{*}$ C'est la preuve qui (en) est qu'il est parti avant midi

77 On peut ainsi retenir que ces SN sous-spécifiés ne fonctionnent pas syntaxiquement comme des Sujets.

\section{2- Quelle est la fonction de la séquence de droite?}

78 Selon plusieurs auteurs (Moreau 1976, Van Peteghem 1991), il apparaît que c'est la séquence postverbale qui est la meilleure candidate à la fonction de Sujet syntaxique. Cette analyse se justifie notamment ${ }^{42}$ par le caractère recevable de :

\section{-l'interrogation en "qu'est-ce qui »:}

[44] Qu'est-ce qui est le problème ? - C'est qu'il est parti avant midi.

- le pseudo-clivage en «ce qui»:

En voici quelques exemples attestés ${ }^{43}$ :

[45] ce qui est le problème, c'est qu'il faut passer par lui pour être tranquille en Tunisie. [web]

[46] Nourrir la population mondiale à 14 milliards d'individus ne serait pas un problème, ce qui est le problème c'est la gestion de tout çà [web]

[47] Ce qui est le problème c'est la cupidité des humains. [web]

[48] Disons que ce qui est le paradoxe, c'est que je suis un des rares directeurs du groupe qui ait une vision de la problématique globale du groupe [web]

Plusieurs attestations, plutôt anciennes toutefois, de ce qui en est la preuve c'est :

[49] Il laisse tout aller à l'aventure; et ce qui en est la preuve, c'est que les gens de bien n'y sont point protégés, ni les méchans punis. [Dassance Pierre, 1837]

[50] Sous ce rapport, nous ferons observer, en terminant, que l'orgueil est le principe du mal ; et, ce qui en est la preuve, c'est qu'il se trouve mêlé aux diverses infirmités de l'âme [Poujoul F., 1857]

- la pronominalisation en "quel » de la séquence postverbale:

Quel est le problème, la conséquence, l'objectif, le motif...

Comme le soutient Van Peteghem $(1991,72)$, « en français on peut reconnaitre le sujet, avec une certitude assez grande, par le fait que, contrairement à l'attribut, il se laisse remplacer par quel ». 


\section{3- Retour au SN sous-spécifié initial : a-t-il une fonction syntaxique?} satisfaisante (ou moins insatisfaisante) que celle en [SVO], il demeure que les $O$ et $S$ présumés ont des particularités telles que plusieurs chercheurs ont choisi de renoncer à retrouver dans ces séquences en $\left[\mathrm{A}\left(\mathrm{c}^{\prime}\right)\right.$ est $\left.\mathrm{B}\right]$ une organisation phrastique canonique qui serait strictement fondée sur des relations morphosyntaxiques, et ont préféré adopter une conception davantage "constructionnelle » mieux à même de rendre compte des spécificités de la structure. C'est ainsi que, pour Blanche-Benveniste (1992, 21), « [i]l semble que l'on ait intérêt à dégager l'originalité de la tournure, en montrant ce qui la fait échapper au classement canonique ». Pour Legallois et Gréa $(2006,167)$ : «plutôt qu’à des règles combinatoires, [l'énoncé spécificationnel] doit sa constitution à une instanciation holistique: il s'agit bien d'un bloc, d'une «tournure toute faite » (...) ». En tant que « tournures originales », c'est aux pseudo-clivées (désormais PCl) que les constructions à Nss ont été le plus régulièrement comparées. Nous allons examiner quels arguments peuvent justifier un tel rapprochement et quelles difficultés demeurent.

\section{b- Constructions spécificationnelles et pseudo-clivage}

94 Les structures à Nss ont pu être mises en relation avec les pseudo-clivées « verbales » canoniques. Par ex. : 
[55] Ce que j'aime c'est qu'il est toujours souriant

L'avantage c'est qu'il est toujours souriant segment gauche y comporte une expression référentielle interprétativement incomplète. (...) Le segment droit a pour effet de saturer pragmatiquement cette simple description en l'unifiant à un référent repéré spatio-temporellement. » Qu'il s'agisse de construction à Nss ou de pseudo-clivée, on relève entre les deux séquences successives une opération de "spécification" (Higgins 1979) qui se réalise d'une manière « progressive » entre le début et la fin de l'énoncé (Roubaud 2000) :

- Dans les PCl, un référent est d'abord introduit de manière peu spécifiée sous forme pronominale dans le segment gauche, puis est développé sous forme lexicale (ou semilexicale) dans le segment droit : ce que $\rightarrow$ il est toujours souriant

- Dans les constructions à Nss, le segment gauche introduit un élément nominal incomplet au plan sémantico-référentiel (Legallois \& Gréa, 2006, 164) et doté d'un caractère abstrait (Schmid 2000,4); le segment droit en fournit une spécification : avantage $\rightarrow$ il est toujours souriant

$\mathrm{Si}$, au vu de telles ressemblances, il apparaît licite de réunir les $\mathrm{PCl}$ prototypiques et les structures à Nss à l'intérieur d'une même « famille » de constructions, la question reste ouverte de savoir si on peut aller jusqu'à les assimiler entièrement. Pour répondre à cette question, tout dépendra évidemment de l'extension que l'on choisira de donner au concept de pseudo-clivage ${ }^{44}$ :

01 Certains travaux vont relativement loin dans la volonté d'assimiler ces deux types: c'est le cas de D. Apothéloz (2012), qui les range sous l'appellation unifiée de " pseudoclivée », en rendant compte de deux sous-types spécifiques. A. Kuyumcuyan $(2018,103)$ prévoit qu'une pseudo-clivée standard puisse avoir un «premier terme nominal». C. Blanche-Benveniste (2010) - tout en insistant sur la nécessité de distinguer plusieurs types syntaxiques - utilise elle-aussi le terme de "pseudo-clivée » de manière plutôt générique, en distinguant entre celles qui manifestent des faits de valence verbale (ce que j'aime c'est...), de valence nominale (le problème c'est...) ${ }^{45}$ et de valence adjectivale (l'ennuyeux c'est...).

D'autres auteurs ne proposent pas d'assimiler entièrement les deux types: c'est le cas de Legallois \& Grea (2006) qui, tout en admettant que «ces constructions sont évidemment à rapprocher des constructions pseudo-clivées » (162-163), distinguent assez nettement « énoncés spécificationnels » et " énoncés pseudo-clivés » (165).

Nous nous rangerons à cette seconde conception ${ }^{46}:$ à nos yeux, le fait que les deux constructions impliquent une prédication spécificationnelle permet certes de les rapprocher sur le plan pragmatique ou sémantique, mais ne les rend pas pour autant équivalents au plan grammatical. Nous nous arrêtons dans la section suivante sur quelques différences entre les deux types. 


\section{1- Distinction entre énoncés à nom sous-spécifiés et pseudo-clivés}

104 Afin de pouvoir donner une justification suffisamment claire de nos positions, il sera utile de revenir en quelques mots sur notre analyse des pseudo-clivées ${ }^{47}$. Dans le cadre méthodologique de l'approche pronominale (Debaisieux dir., 2013), un certain nombre d'énoncés dans lesquels c'est possède un statut «affaibli », ont été analysés comme des « dispositifs de la rection»:

Par exemple, dans les énoncés clivés (type c'est Marie qui a répondu), nous renonçons à décrire la forme c'est comme le verbe recteur de la construction, et Marie qui a répondu comme un SN à relative. En effet, cette façon de voir poserait de sérieux problèmes, en particulier lorsque l'élément touché par l'opération de clivage concerne une autre catégorie que le nom : par exemple, dans c'est demain qu'il va venir, il serait difficile de reconnaitre dans la séquence demain qu'il va venir une suite [antécédent + relative]. Nous dirons donc que, dans ce dernier exemple, c'est venir qui est le verbe recteur, alors que demain et il sont respectivement un Ajout et le Sujet de ce verbe. Quant à c'est, il a ici le statut d'un «auxiliaire de dispositif » qui introduit l'élément clivé en le dotant d'une valeur focalisante.

De la même manière, la forme c'est des énoncés $\mathrm{PCl}$ prototypiques constitue un auxiliaire de dispositif qui n'a, en tant que tel, aucun statut au plan des relations de dépendances microsyntaxiques engagées dans la construction : dans un énoncé comme ce que je lui reproche c'est de ne pas être resté, la forme c'est ne sélectionne aucune valence propre. C'est le verbe reprocher qui, en tant qu'élément recteur, est responsable de la sélection de son Objet Direct (donné sous forme non spécifiée d'abord - ce que - puis spécifiée en fin de structure - de ne pas être resté), de son Sujet (je) et de son objet Indirect (lui).

107 Sur la base de cette présentation, nous nous demanderons dans quelle mesure les énoncés à Nss peuvent être assimilés au dispositif pseudo-clivét8.

\section{- Forme de réalisation de être}

Les propositions qui visent à assimiler constructions à Nss et $\mathrm{PCl}$ impliquent que le verbe être aurait exactement le même statut de "verbe copule» ou de "copule équative $»^{49}$ dans les deux cas. Or certaines différences peuvent être relevées :

$110-$ - Est » ou «c'est»?

111 Dans les pseudo-clivées, il est généralement admis que la forme c'est, avec réalisation de l'élément pronominal, est la forme non marquée, qui est de fait très largement majoritaire dans les corpus, par ex. :

[56] ce qu'ils attendent de leur mutuelle, c'est d'abord une aide pour financer leurs études, se loger, et aussi une assurance automobile. [ex. Roubaud, 2000, p. 287]

112 Grevisse (1964) $)^{50}$ notait déjà que dans ces constructions, la « reprise du Sujet " par ce semblait obligatoire. Selon Valli $(1981,197)$ la libre alternance entre est et c'est a sans doute existé jusqu'à l'époque classique mais semble devenue marginale dans le français contemporain.

113 A l'inverse, le être des constructions à Nss se rencontre de manière tout à fait naturelle avec ou sans le c' : Le problème est que... / Le problème c'est que...

114 Comme le précise C. Blanche-Benveniste $(1992,2)$, « dans le français de conversation, on trouve plus souvent la forme c'est que le forme est, bien que les deux paraissent partout interchangeables $»^{51}$. 
Il apparaît en effet que, tout au moins dans les corpus écrits consultés, les constructions à Nss dont la partie droite est constituée par une Que-C. sont attestées dans les deux versions ; sur une sélection de 5 lexèmes ${ }^{52}$, la base FWC révèle la répartition suivante :

\begin{tabular}{|l|c|c|}
\hline & est / était + Que-C. & c'est / c'était +Que-C. \\
\hline La preuve & 2211 & 1625 \\
\hline Le fait & Très majoritaire & Très minoritaire \\
\hline Le problème & 33359 & 35884 \\
\hline Le souci & 2249 & 625 \\
\hline La raison & 3151 & 548 \\
\hline
\end{tabular}

Pour les constructions introduisant les lexèmes problème et souci et dont la séquence droite est constituée par un groupe en de + Inf., la répartition est la suivante :

\begin{tabular}{|l|c|c|}
\hline & Est / était $+d e$ Inf & C'est / c'était $+d e$ Inf \\
\hline Le problème & 4968 & 785 \\
\hline Le souci & 476 & 123 \\
\hline
\end{tabular}

Ces résultats, qu'il conviendrait de vérifier sur d'autres lexèmes et sur d'autres types de corpus, suffisent à montrer que les deux versions, avec ou sans $c^{\prime}$, sont à chaque fois attestées, et que les réalisations sans pronom sont assez nettement majoritaires à l'écrit ${ }^{53}$.

118 On conclura que cette possibilité d'alternance semble faire de la copule un verbe somme toute assez «ordinaire » quant à sa possibilité de réaliser ou non un double marquage sur le Sujet (cf. ton père a raison / ton père il a raison).

- Variation temporelle de "être»

Comme l'avait noté Valli (1981) et comme le soulignent Apothéloz \& Roubaud $(2015,3)$, "dans les pseudo-clivées, le verbe être présente des capacités réduites à choisir ses temps, modes et aspects [...]. Dans la très grande majorité des exemples, le verbe être est au présent et au singulier, ce qui indique une forte tendance à l'invariabilité ». En particulier, C. Blanche-Benveniste (2002-a) notait que les formes auxiliées (a été, avait été...) sont très peu attestées dans les pseudo-clivées et les autres dispositifs de la rection.

121 C'est donc invariablement le verbe recteur situé dans la séquence de gauche qui porte les marques temporelles / aspectuelles (affixe flexionnel, ou verbe auxiliaire ou semiauxiliaire), alors que l'auxiliaire de dispositif c'est tend à se maintenir au présent :

[57] ce que je veux / voulais / voudrais / aurais voulu c'est qu'il vienne

Dans les constructions à Nss, la forme de être est manifestement moins bloquée à cet égard ${ }^{54}$. Nous ne sommes pas en mesure de donner d'estimation quantitative précise, mais les quelques exemples attestés suivants, qui proviennent du web, illustrent le caractère tout à fait naturel de la variation temporelle de être, y compris pour ce qui concerne les formes auxiliées (a été, avait été, aurait été) :

[58] L'avantage a été que nous n'avons jamais perdu notre objectif de vue

[59] Le souci a été que les casques et les micros prenaient aussi la poussière ambiante.

[60] Mon problème, ça a été de choisir l'ordre des poèmes dans cette anthologie 
[61] Notre problème avait été que la femme de ménage avait fait tombé une serpillère dans les toilettes.

[62] Le problème avait été que Lehman Brothers avait été autorisée à réussir

[63] l'avantage ça aurait été que si il y a interruption du service (coucou la wifi sur DS :) ), il sera toujours possible de transférer ses pokémon.

[64] Comment faire? car meme si je la rabote le souci va etre que l'air va passer!!!

Autre fait remarquable : la disponibilité de la forme en est, non précédée du pronom, autorise l'emploi du participe présent (dont nous avons vu plus haut qu'il était bien attesté dans les constructions en est c'est). Voir ces ex. en l'avantage / le souci étant :

[65] Grâce à cette application [...], il est possible d'obtenir un tableau nutritionnel complet du produit que l'on achète, d'explorer ses additifs, ses éventuels allergènes, ses labels, ses informations de traçabilité, son empreinte carbone... L'avantage étant que l'on peut contribuer à cette base de données. [web]

[66] La situation est la suivante j'ai commander a la date du 11 juin trois articles que j'ai payé avec une carte cadeaux carrefour, le souci etant que l'un des articles n'etant disponible que fin juillet, j'ai pris la decision de d'annuler cet article de ma commande par le biais du service client. [web]

Dans le cas des PCl en c'est, la chose serait évidemment impossible :

[67] * Ce que mon père aime c'étant le cinéma scandinave

Et même en laissant tomber le pronom $c^{\prime}$, l'exemple reste très artificiel :

[68] ? Ce que mon père aime étant le cinéma scandinave, nous avons surtout regardé des films de Bergman.

On pourra retenir de ces quelques caractéristiques que la copule des constructions à Nss possède un degré de figement bien moindre que ce n'est le cas du c'est de dispositif tel qu'il apparaît dans les pseudo-clivées: on constate pour ces premières une alternance entre les formes à pronom (c'est) et celles sans pronom (est), de même qu'une variabilité dans le choix du temps verbal. Pour paraphraser C. BlancheBenveniste (2002-a), ces faits suggèrent que le être des constructions à Nss possède un "degré de verbalité » bien plus important que l'auxiliaire de dispositif c'est présent dans les $\mathrm{PCl}$, qui est en comparaison particulièrement amorphe au plan morphosyntaxique. Si les constructions à Nss étaient largement identifiables aux pseudo-clivées, on voit mal pourquoi la forme de réalisation de être connaitrait une différence aussi marquée.

On rappelle que dans les $\mathrm{PCl}$ prototypiques, la séquence droite est régie par un élément recteur - en l'occurrence un verbe - apparaissant dans le segment gauche. Ce lien peut se vérifier par l'attestation du passage à la forme directe qui, si elle n'est pas systématique, est régulièrement attestée :

[69] ce que j'aime c'est [le cinéma]o $\rightarrow$ j'aime [le cinéma]o

celui qui a parlé c'est [Paul] $\rightarrow$ [Paul] $S$ a parlé

Il apparait ainsi que les propriétés syntaxiques des pseudo-clivées restent, sur le plan dépendanciel, assez « ordinaires » au sens où l'élément extrait (le cinéma, Paul) exerce une fonction syntaxique tout à fait canonique (Objet, Sujet) auprès de son verbe recteur (aimer, parler).

130 Nous allons montrer, à partir des lexèmes inconvénient et preuve, que cette caractéristique ne se retrouve pas dans les constructions spécificationnelles à Nss. 
On peut observer que ce lexème, dans ses emplois usuels, sous-catégorise, entre autres « compléments « ou « modifieurs » du nom, des SP en de ou à ; par exemple :

[70] L'inconvénient de cette méthode

d'être auto-entrepreneur

à ce type de traitement

à utiliser un hélicoptère

On vérifie le caractère régi du SP en relevant que l'ensemble du grand constituant formé par [Det + Nom + SP] se retrouverait sans difficulté doté d'une syntaxe externe, soit comme Objet de Verbe (Il faudra évoquer l'inconvénient d'être auto-entrepreneur / ... l'inconvénient à ce type de traitement...), soit comme Sujet (l'inconvénient d'être autoentrepreneur... / l'inconvénient à ce type de traitement ne doit pas être sous-estimé).

Il est frappant de constater que cette relation de rection entre le nom et le SP ne peut pas s'actualiser sous la forme d'une structure à Nss :

Soit on obtient des formes carrément agrammaticales :

[71] *l'inconvénient (c') est [de cette méthode]

*L'inconvénient ( $\left.c^{\prime}\right)$ est [à ce type de traitement]

*L'inconvénient (c') est [à utiliser un hélicoptère]

Soit on obtient un énoncé effectivement envisageable :

[72] L'inconvénient (c') est [d'être auto-entrepreneur],

mais dans lequel le SP placé après la copule semble difficile à analyser comme un modifieur du nom inconvénient, et cela pour les deux raisons suivantes :

- D'une part, on relève que la séquence d'être auto-entrepreneur pourrait facilement demeurer auprès de son nom recteur, et la partie droite de la structure être instanciée par un autre élément, un autre SP par exemple :

[73] Le principal inconvénient d'être auto-entrepreneur (c')est de ne pas avoir

d'assurance-chômage

En ce cas, la position syntaxique de complément du nom étant saturée dès la première séquence de la construction à Nss, il est difficile de donner au SP de droite le statut d'un élément régi par le nom.

140 - D'autre part, dans le cas d'un SP régi par un nom, on constate pour d'assez nombreux lexèmes un phénomène d'alternance assez régulier entre les SP de type «de + V.inf » et ceux de type «de $+\mathrm{SN} »$ : par ex. :

[74] L'inconvénient d'être auto-entrepreneur / du statut d'auto-entrepreneur

Le choix de faire le bien / de la philanthropie

Ma crainte de me retrouver seul / de la solitude

Dans les constructions à Nss, une telle alternance ne se vérifie nullement; on pourra avoir la suite de + V.inf. :

[75] L'inconvénient (c') est d'être auto-entrepreneur

Le meilleur choix serait de faire le bien

Ma crainte était de me retrouver seul

Mais jamais la suite de $+\mathrm{SN}$ :

[76] *'inconvénient (c') est du statut d'auto-entrepreneur

*Le meilleur choix serait de la philanthropie

* Ma crainte était de la solitude

Ces propriétés illustrent le fait que, contrairement à ce que les apparences pourraient suggérer, on ne peut pas accorder le même statut syntaxique aux séquences en de Inf. 
qui sont contiguës au nom et à celles qui apparaissent en deuxième partie de construction à Nss. - du nom preuve.

[82] la preuve (c') est [qu'il est coupable]

en proposant d'analyser dans les deux cas la Que-C. comme complément - ou modifieur

Mais, ici encore, une telle analyse pose problème : il apparaît en fait que la Que-C. régie qu'il est coupable pourrait aisément rester auprès de son nom recteur, alors que la partie droite de la structure spécificationnelle pourrait être instanciée par une autre Que-C :

[83] [la preuve [qu'il est coupable $]_{\text {régi }}$ ] c'est [qu'il n'a pas voulu faire appel]

Or dès que la Que-C. est placée dans le segment droit, elle n'a plus aucune alternance possible avec un SP comme de son refus de faire appel, qui est pourtant bien souscatégorisé par le nom preuve:

[84] *[la preuve [qu'il est coupable $]_{\text {régil }}$ c'est [de son refus de faire faire appel]

Ceci rend extrêmement difficile la description des Que-C. apparaissant après la copule comme des faits de dépendance nominale, même dans le cas des noms comme preuve, qui ont la capacité de régir une Que-C. ${ }^{55}$

4 A l'inverse du nom preuve, il est manifeste que de nombreux noms sous-spécifiés qui, a priori, ne régissent pas de Que-C. pourraient être employés dans des constructions spécificationnelles terminées par une Que-C. :

[85] ? la solution qu'il parle en anglais

la solution ce serait qu'il parle en anglais

[86] ? le hic qu'il est arrivé en retard

le hic c'est qu'il est arrivé en retard

[87] ? le mystère qu'il n'est / ne soit jamais revenu

le mystère est qu'il n'est / ne soit jamais revenu

Ces phénomènes confirment qu'en de nombreux cas $^{56}$, la relation entre un nom sousspécifié et la séquence qui apporte la spécification n'a pas les caractéristiques d'une 
relation de dépendance syntaxique ordinaire entre un nom recteur et son modifieur, mais manifeste un autre type de relation, essentiellement sémantique.

\section{c- Sur l'analyse grammaticale des constructions à Nss : éléments de synthèse}

Comme nous l'avons rappelé dans les deux sections précédentes, la description grammaticale des constructions spécificationnelles à Nss se heurte à une double difficulté :

157 - La description qui fait de être un verbe régissant un Sujet et un Objet/Attribut présente des limites connues depuis longtemps: si dans un exemple tel que la conséquence sera qu'on ne le verra plus, la séquence de droite peut à plusieurs égards être considérée comme un type de Sujet, il semble en revanche impossible de conférer une fonction syntaxique au SN comportant le nom sous-spécifié (la conséquence), dont le caractère non délexicalisable, non clivable et non contrastable interdit, dans notre perspective, d'en faire un élément gouverné par le verbe.

158 - L'analyse qui voudrait assimiler les constructions spécificationnelles à Nss et les pseudo-clivées présente également des faiblesses : d'une part, le verbe être ne peut pas être considéré comme un auxiliaire de dispositif semblable à celui qu'on trouverait dans une $\mathrm{PCl}$ prototypique (en raison des variations formelles avec lesquelles il est compatible), mais reste à plusieurs égards proche d'un verbe copule ordinaire. D'autre part, la relation qui s'instaure entre le nom sous-spécifié et la séquence spécifiante postverbale ne peut pas - dans de nombreux cas du moins - être définie comme une relation de dépendance syntaxique du même type que celle qui s'exercerait entre le verbe et sa rection à l'intérieur d'une $\mathrm{PCl}$ canonique.

De cette double difficulté nait un double sentiment :

160 - D'une part, vu que le SN initial des constructions à Nss ne régit pas la séquence spécifiante postverbale, il faut admettre que c'est bien la copule qui joue son rôle dans la sélection de la séquence en question. La chose peut être illustrée par un exemple comme :

[88] La conséquence sera qu'on ne le verra plus

161 On note que, dans une structure purement parataxique, dans laquelle le verbe être serait absent, le morphème que serait absent lui aussi, ce qui montre que ce dernier est formellement apporté par la copule :

[89] Conséquence : on ne le verra plus !

? Conséquence : qu'on ne le verra plus.

162 - Mais, par ailleurs, il existe indiscutablement des préférences sélectionnelles entre le lexème sous-spécifié et la catégorie de la séquence spécifiante (cf. Legallois, 2008) : par exemple, le nom question sera préférentiellement lié à une séquence en de Inf (la question est de...) mais le nom hic déclenchera plutôt une Que-C. (le hic est que...). Schmid $(2018,117-118)$ y voit essentiellement une question de compatibilité sémantique entre le nom sous-spécifié et la séquence spécifiante : " to-infinitives are selected for the complementation of nouns encoding aspects such as 'manipulation' [...], 'wanting' [...] or the so-called "de re" domain of actions and events [...], while that-clauses complement nouns encoding aspects such as 'knowing', 'believing', 'saying' or the 'de dicto' domain." 

forme de être : soit bas

Il semble donc évident que le sémantisme du nom sous-spécifié contribue à orienter la catégorie de la séquence postverbale.

En raison de ce double jeu d'influence grammaticale - celle du nom sous-spécifié et celle de la copule, on pourrait presque considérer que l'élément recteur de ces structures ne serait en fin de compte ni la copule à elle seule ni le SN en tant que tel, mais un élément recteur "complexe», composé de l'ensemble formé par le SN et la

[90] [La conséquence sera $]_{\text {recteur }}$ qu'on ne le verra plus

Dans une telle conception, et conformément à la présentation faite plus haut au point C.1.a., on pourrait admettre en outre que cet élément « recteur complexe » gouverne un constituant Sujet postposé :

[91] [La conséquence sera] $]_{\text {recteur }}$ [qu'on ne le verra plus]S

La reconnaissance que la suite [Nom + verbe] formerait un ensemble recteur (qui n'est posée qu'à titre d'hypothèse ici) serait évidemment compatible avec les approches phraséologiques proposant de concevoir les structures spécificationnelles comme des unités préconstruites (Legallois \& Grea, 2006).

Sur la base de cette proposition, revenons à présent aux formes non prototypiques en est c'est, qui ont été illustrées dans la première partie de cet article.

\section{2- Les constructions spécificationnelles en est c'est}

Pour rendre compte d'un énoncé comme [18], partiellement reproduit ici :

[18] le problème est c'est que les consommateurs souhaitent que le prix de vente

nous ne ferons pas l'hypothèse qu'on aurait affaire à une pseudo-clivée elliptique, qui pourrait être mise en relation avec (ce que) le problème est, c'est que... ${ }^{57}$

Nous ne ferons pas non plus l'hypothèse que la structure illustrerait un pur phénomène de duplication comme le font certains linguistes qui décrivent les structures en is is de l'anglais comme des "reduplicative copulas $»^{58}$. L'idée de duplication nous parait gênante, pour le cas du français, dans la mesure où elle semble suggérer que les formes verbales successives auraient le même statut grammatical et feraient l'objet d'une simple réitération. Loin de se limiter à un phénomène de duplication, l'originalité de la tournure réside selon nous dans le fait qu'elle amalgame ${ }^{59}$, ou superpose, deux occurrences de être qui relèvent de fonctionnements distincts.

1 Nous proposons de schématiser ci-dessous l'organisation des énoncés spécificationnels en prévoyant une zone distincte pour chacun des deux emplois de être. Les deux premières lignes présentent la structure des énoncés prototypiques; les formes en est $c^{\prime} e s t$ sont indiquées sur la dernière ligne du tableau ${ }^{60}$ : 


\begin{tabular}{|c|c|c|c|}
\hline Nss & $\begin{array}{l}\text { Zone de être } \\
\text { en emploi } \\
\text { copulatif }\end{array}$ & $\begin{array}{l}\text { Zone de c'est } \\
\text { auxiliaire de } \\
\text { dispositif }\end{array}$ & $\begin{array}{l}\text { Sujet du recteur } \\
\text { complexe [Nss est] }\end{array}$ \\
\hline Le problème & $\begin{array}{l}\text { est } \\
\text { était } \\
\text { a été } \\
\text { sera } \\
\text { étant }\end{array}$ & & $\begin{array}{l}\text { Que-C. } \\
\text { de Inf } \\
\text { SN }\end{array}$ \\
\hline Le problème & $\begin{array}{l}\text { c'est } \\
\text { ça a été } \\
\text { c'était } \\
\text { ce / ça sera } \\
* \text { c'étant }\end{array}$ & & $\begin{array}{l}\text { Que-C. } \\
\text { de Inf } \\
\text { SN }\end{array}$ \\
\hline Le problème & $\begin{array}{l}\text { est } \\
\text { a été } \\
\text { était } \\
\text { sera } \\
\text { étant }\end{array}$ & c'est & $\begin{array}{l}\text { Que-C. } \\
\text { de Inf } \\
\text { SN }\end{array}$ \\
\hline
\end{tabular}
(le problème est), soit le marquage informationnel (le problème, c'est). Il faut donc faire l'hypothèse que, chez certains locuteurs et pour certains types de lexèmes sousspécifiés, la stratégie a ou b prise isolément est moins satisfaisante que l'amalgame des deux stratégies $\mathrm{a}+\mathrm{b}:$ le problème est c'est. Toute la question est de savoir pour quelles raisons une séquence comme le problème est c'est de l'énoncé [18] ci-dessus se trouverait préférée aux alternatives plus normatives. 
179 En fin de compte, dans les constructions en est c'est, ce n'est pas la présence de la forme c'est qui est la plus intrigante : on sait en effet que, au-delà des seules clivées et pseudoclivées, le français a une forte tendance à employer ce type de marqueur dans les énoncés parataxiques «à effet deux points » du type :j'ai un souci, c'est qu'il est parti / il y a quelque chose, c'est qu'on a oublié la clé...). Comme cela a souvent été relevé (Apothéloz 2008), l'usage du marqueur c'est, et l'effet de binarisation macrosyntaxique qu'il produit, est très caractéristique des énoncés spécificationnels au sens large ${ }^{61}$.

180 C'est davantage le fait de conserver la séquence contiguë [le Nss+est] qui a de quoi surprendre. En effet, cela a pour résultat la formation d'une séquence Prénoyau tout à fait atypique. Contrairement aux Prénoyaux canoniques, qui, par exemple, prendraient la forme d'une construction nominale (type le problème) ou verbale (type on a un problème), le Prénoyau des énoncés en est c'est aura une forme comme le problème est, qui semble irrégulière au regard des règles de constituance syntaxique :

\begin{tabular}{|l|l|}
\hline Le problème & c'est qu'il est parti \\
\hline $\begin{array}{l}\text { Il y a un problème } \\
\text { On a un problème }\end{array}$ & \\
\hline Le problème est & \\
\hline [ PRÉNOYAU ] & [ NOYAU ] \\
\hline
\end{tabular}


Les possibilités d'autonomisation de la séquence vont même plus loin puisqu'on peut aussi la trouver comme énoncé indépendant :

[94] Et l'un a piqué la femme de l'autre- bien sûr ça ne se disait pas comme ça à l'époque ! Mais le fait est. Cependant, pas de problème, ils sont restés amis et d'ailleurs ils vivent encore ensemble. (Linhart N., Le jour où mon père s'est tu, 2008)

b- Le nom preuve est de loin le lexème le plus fréquent dans notre corpus d'énoncés en est c'est. Il est intéressant de voir que c'est la forme combinée au clitique en qui est la mieux attestée dans les cas où le verbe être est au présent : la preuve en est c'est que... (cf. [2] et [10]). En examinant sa syntaxe interne, il est possible de montrer qu'une séquence comme la preuve en est tient de la "formule toute faite » : la présence du clitique en est à elle seule révélatrice; on sait en effet que, dans la langue ordinaire, l'emploi d'un tel pronom comme élément de rection nominale est extrêmement rare ${ }^{63}$. Des exemples tels que les suivants sont très peu susceptibles d'être recueillis dans des corpus de français parlé informel :

[95] J'ai vu ce film. Le dénouement en est bouleversant [en = de ce film]

Ce bâtiment vient d'être rénové. Toute la toiture en a été refaite [en = de ce bâtiment]

Si bien que la haute fréquence de l'association entre le nom et le clitique dans la preuve en est paraît symptomatique du caractère vraisemblablement préconstruit de cette forme.

Pour ce qui concerne son intégration à l'intérieur des énoncés, on peut noter que cette expression (avec ou sans la présence du Déterminant) est susceptible - tout comme le fait est - de s'autonomiser en tant que constituant détaché en début d'énoncé, dans des organisations reposant sur les liens de parataxe :

[96] Je n'ai pas voulu résister, je ne l'ai pas voulu et ne croyez pas que j'ai résisté, la preuve en est : j'ai donné mon frère et ma maîtresse ! (G. Bataille, L'abbé C. 1950)

[97] Depuis des années, les animaux de compagnie ont le vent en poupe auprès des Français [...]. Preuve en est, il y a quasiment autant d'animaux de compagnie dans l'Hexagone que d'habitants : 63 millions ! [web]

Qui plus est, cette séquence semble pouvoir aller, chez certains locuteurs en tout cas, jusqu'à former un énoncé autonome : (La) preuve en est !

Il semblerait ainsi que le caractère fortement figé des suites le fait est et la preuve en est ainsi que leur possibilité d'autonomisation macrosyntaxique contribuent à favoriser leur apparition dans les structures du type le fait est c'est que..., et la preuve en est c'est que...

Les choses sont moins évidentes pour d'autres séquences bien attestées dans les structures en est c'est, mais dont le caractère locutionnel nous semble moins manifeste : le problème est et le souci est.

A propos du nom problème, on relève qu'il intervient très fréquemment dans les constructions spécificationnelles canoniques : dans leur corpus d'écrit journalistique, Legallois \& Gréa $(2006,175)$ relèvent que $30 \%$ de la totalité des emplois concernent le nom problème: «Problème est Que-P. est donc fortement enraciné, implanté dans notre compétence discursive, qui se nourrit, non pas (seulement) de règles syntaxiques, mais de l'ensemble des occurrences rencontrées dans l'expérience discursive » (176). Mis à part les arguments quantitatifs attestant de la fréquence de la tournure, il est plus difficile que pour les noms preuve ou fait de démontrer le caractère préconstruit des séquences en le problème est. On relève tout de même dans le web des exemples dans 
lesquels des séquences de ce type constituent le premier segment d'énoncés binaires articulés sur un mode parataxique :

[98] Un nouveau collaborateur est arrivé au bureau depuis plusieurs mois. Rien à signaler, il est plutôt sympa et travailleur. Le problème est je constate sans cesse qu'il me regarde avec insistance. [web]

[99] Je l'ai redémarré plusieurs fois, et même réinitialisé les paramètres réseau, réinitialisé les apn, vérifiée les réseaux, le problème est : je suis en débit limité [web] [100] Dois-je laisser la filtration enclenchée au maximum pour garder une eau claire ? le probleme est elle s'éteint automatiquement [web]

A noter que le nom souci, bien représenté dans notre corpus d'énoncés en est c'est, entre dans des organisations semblables :

[101] Ca fait plaisir de voir qu'on n'est pas seuls ! Le souci est...je passe mon permis dans 4 mois ! Et je ne peux toujours pas conduire plus de $10 \mathrm{~km}$ sur l'autoroute en étant "à l'aise". [web]

[102] Mon souci est : elle démarre mal à chaud, il faut tirer pas mal sur la batterie.

Celle ci est neuve, comme les bougies. [web]

Ici encore, on pourrait donc faire l'hypothèse que la relative "disponibilité » de séquences comme le problème est ou le souci est contribuent à faciliter leur insertion dans les énoncés non prototypiques en est c'est.

\section{CONCLUSION}

Les énoncés spécificationnels à nom sous-spécifié du français peuvent se réaliser sous une forme non prototypique qui réalise deux fois le verbe être : le problème est c'est... Les données indiquent que ces constructions sont peu fréquentes; elles semblent toutefois suffisamment bien attestées, notamment avec certains lexèmes, pour qu'on puisse y voir une structure grammaticale et non la simple manifestation d'" accidents" de performance. Au plan grammatical, nous avons proposé de les analyser comme des structures qui superposent deux emplois distincts du verbe être: d'abord, un emploi copulatif dans lequel la forme verbale a la particularité de pouvoir varier en temps (est, était, étant...) ; en second lieu, la forme c'est, à laquelle nous accordons la valeur d'un auxiliaire de dispositif, comme on en trouverait dans les énoncés clivés ou pseudoclivés. La réalisation de tels énoncés résulte d'une tension entre deux stratégies antagonistes : celle visant à conserver la forme grammaticale la plus canonique dans laquelle le nom sous-spécifié et le verbe copulatif sont strictement contigus; et celle visant à souligner la stratification informationnelle des énoncés en topic/focus par l'usage du pronom de reprise (c').

De manière incidente, l'examen de notre corpus nous a conduit à vérifier que la forme c'est des structure pseudo-clivées et la forme (c')est des constructions spécificationnelles à nom sous-spécifié ne pouvaient pas être assimilées dans un emploi "copulatif " uniforme mais gagnaient au contraire à être distinguées. 


\section{BIBLIOGRAPHIE}

Andersen, G. (2002). “Corpora and the Double Copula”. From the COLT's mouth... and others: Language Corpora Studies, in Honor of Anna-Brita Strenstrom. Amsterdam : Rodopi, 43-58.

Apotheloz, D. (2008). «A l'interface du système linguistique et du discours : l'exemple des constructions identificatives (e.g. pseudo-clivées) ». In O. Bertrand, S. Prévost, M. Charolles, J. François \& C. Schnedecker (éds), Discours, diachronie, stylistique du français. Études en hommage à Bernard Combettes. Berne : Peter Lang, 75-92.

Apothéloz, D. (2012). «Pseudo-clivées et constructions apparentées ». In Groupe de Fribourg, Grammaire de la période. Berne : Peter Lang, 207-232.

Apothéloz, D. (2018). « Examen d'une famille de constructions : les constructions identificatives ». SCOLIA 32, 13-41.

Apotheloz, D. \& Roubaud M.-N. (2015). « Constructions pseudo-clivées ». Encyclopédie Grammaticale du Français, http://encyclogram.fr

Blanche-Benveniste, C. (1990). "Grammaire première et grammaire seconde : l'exemple de en ». Recherches sur le français parlé 10, 51-73.

Blanche-Benveniste, C. (1992). «Sur un type de nom 'évaluatif' portant sur des séquences verbales ». I.T.L. Review of Applied Lingustics 97-98, 1-25.

Blanche-Benveniste, C. (2002-a). « Auxiliaires et degrés de 'verbalité' ». Syntaxe et sémantique 2002/1 (N³), 75-97.

Blanche-Benveniste, C. (2002-b). « Macro-syntaxe et micro-syntaxe : les dispositifs de la rection verbale ». In H.L. Andersen \& H. Nølke (éds.), Macro-syntaxe et macro-sémantique. Berne : Peter Lang, 95-115.

Blanche-Benveniste, C. (2010). « Les pseudo-clivées et l'effet deux points ». In M.-J. Béguelin, M. Avanzi \& G. Corminboeuf (éds), La Parataxe, t. 2, Berne : Peter Lang, 185-217.

Bolinger, D. (1987). “The Remarkable Double Is”. English Today 9, 39-40.

Brenier, J.M. \& Michaelis, L. (2005). « Optimization via syntactic amalgam: syntax-prosody mismatch and copula doubling". Corpus Linguistics and Linguistic Theory 1, 1, 45-88.

Coppock, E., Brenier, J., Michaelis, L. \& Staum L. (2006). “ISIS: It's not a disfluency, but how do we know that?". Paper presented at the 32nd Annual Meeting of the Berkeley Linguistic Society. Berkeley, CA, 85-96.

Debaisieux, J.-M. (dir.) (2013). Analyses linguistiques sur corpus. Subordination et insubordination en français. Paris : Lavoisier / Hermes Sciences.

Higgins, F. R. (1979). The Pseudo-cleft Construction in English. New York : Garland Publishing. Jakubíček, M., Kilgarriff, A., Kovář, V., Rychlý, P. \& Suchomel, V. (2013). “The TenTen corpus family". Proc. Int. Conf. on Corpus Linguistics, Lancaster.

Keizer, E. (2013). “The X is (is) construction”. In J.L. Mackenzie \& H. Olbertz (éds.), Casebook in Functional Discourse Grammar. Amsterdan : John Benjamins, 213-248.

Kuyumcuyan, A. (2018). «L'extension des pseudo-clivées ». SCOLIA 32, 95-116. 
Lambrecht, K. \& Ross-Hagebaum, S. (2006). “Apo Koinou and Intrusion: Toward a SyntacticPragmatic Typology of Amalgam Constructions in Spoken English”. 4th Workshop on Discourse Structure, UT Austin. March 3-5.

Legallois, D. (2006). «Quand le texte signale sa structure : la fonction textuelle des noms sousspécifiés ». CORELA, HS-5 Organisation des textes et cohérence des discours.

Legallois, D. (2008). « Sur quelques caractéristiques des noms sous-spécifiés ». Scolia 23, 109-127.

Legallois, D. \& Grea, P. (2006). « L'objectif de cet article est de... Construction spécificationnelle et grammaire phraséologique ». Cahiers de Praxématique 46, 161-186.

Lenepveu, V. (2009). «A propos de l'expression nominale le fait est que ». Le français moderne LXXVII, 2, 199-218.

Martinie, B. \& Sitri, F. (1999). « Insertion des deux points dans des structures bisegmentales : les limites de la thématisation ». In C. Guimier (éd.) La thématisation dans les langues. Berne : Peter Lang, 151-168.

Massam, D. (1999). “Thing is constructions: the thing is, is what's the right analysis?”. English Language and Linguistics 3(2), 335-352.

Moreau, M.-L. (1976). Etude de syntaxe transformationnelle. Univ. de Mons.

O'Neill, T. (2014). “Demystifying double-is”. Talk handout, CUNY Graduate Center, [https:// ygdp.yale.edu/phenomena/double-is].

Roubaud, M.-N. (2000). Les constructions pseudo-clivées en français contemporain. Paris : Champion.

Roubaud, M.-N. \& Sabio, F. (2018). «Ce qu'il y a c'est que mon portefeuille il est pas extensible : 'il y a' et le pseudo-clivage ». SCOLIA 32, 43-67.

Rouveret, A. (1998). « Points de vue sur le verbe 'être' ». In A. Rouveret (éd.), 'Être' et 'avoir'. Syntaxe, sémantique, typologie. Presses Universitaires de Vincennes, 11-65.

Sabio, F. \& Benzitoun, C. (2013). «Sur les relations entre syntaxe et discours : dispositifs de la rection et dispositifs macrosyntaxiques ». Studia Universitatis Babes-Bolyai Philologia, vol.58 (LVIII), $4,97-110$.

Schmid, H.-J. (2000). English abstract nouns as conceptual shells: from corpus to cognition. Berlin / New York : Mouton de Gruyter.

Schmid, H.-J. (2018). “Shell nouns in English. A personal roundup”. Cappletra 64, 109-128.

Shapiro, M \& Haley, M. (2002). "The reduplicative copula is is". American Speech 77(3), 305-312.

Teston-Bonnard, S. (2018). Particules discursives verbales et constructions concises verbales : un continuum de propriétés ou deux catégories bien différentes? Aspects syntaxiques et pragmatiques. Mémoire HDR.

Tuggy, D. (1995). “The thing is is that people talk that way. The question is why?". In E. H. Casad (éd.) Cognitive Linguistics in the Redwoods: The Expansion of a New Paradigm in Linguistics. Berlin : Mouton de Gruyter, 713-752.

Valli, A. (1981). « Notes sur les constructions dites 'pseudo-clivées' en français ». Recherches sur le français parlé 3, 195-211.

Van Peteghem, M. (1991). Les phrases copulatives dans les langues romanes. Wilhelmsfeld : Gottfried Egert Verlag. 
Yale Grammatical Diversity Project - English in North America- Double is [https://

ygdp.yale.edu/phenomena/double-is]

\section{NOTES}

1. http://alerte-environnement.fr/2015/09/03/crise-agricole-debloquons-linnovation/

2. https://www.lequipe.fr/Football/Actualites/Lyon-comment-mariano-diaz-est-devenu-pluscollectif/850750

http://the-temple-of-whiskers.over-blog.com/2015/03/le-hobbit-dans-la-mire-duhunter.html

4. Nous adoptons la désignation de Legallois (2008), qui reprend le terme de "spécification » à Higgins (1979). Apothéloz $(2008,2018)$ emploie le terme de « constructions identificatives ».

5. Est-ce parce qu'ils sont trop peu fréquents pour avoir retenu l'attention des puristes? Nous n'avons pas trouvé de condamnation de ces tours dans les ouvrages normatifs usuels, à l'exception d'un blog consacré à la langue française qui dénonce une « incorrection patente » [La grammaire de Forator: http://la-grammaire-de-forator.over-blog.fr/2015/04/le-fait-est-c-estque.html]

6. https://www.troc-velo.com/index.php?forum=1\&cmd=viewpost\&idf=1\&idp=26049

7. Dont nous avons exploité une sous-partie, celle des sites dont l'extension est en « fr ».

8. https://www.sketchengine.eu/

9. Nous ne reviendrons pas ici sur la définition des noms sous-spécifiés; on les caractérise usuellement par une double propriété : sémantique d'abord (comme le précise Legallois, 2006, « ces noms relativement 'abstraits' [...] ne donnent pas naissance à des représentations précises ou stables des objets auxquels ils renvoient virtuellement»); syntaxique ensuite (leur prédisposition à «encapsuler » un contenu propositionnel les rend apte à entrer dans des structures spécificationnelles, du type « $\mathrm{N}$ est Que-C.» ou « $\mathrm{N}$ est de Inf »). Or chacun peut observer que ces deux dimensions ne sont pas strictement congruentes l'une à l'autre : tous les lexèmes «abstraits » n'entrent pas nécessairement dans des structures spécificationnelles (Schmidt, 2018, 111). A l'inverse, certains lexèmes sont compatibles avec les constructions spécificationnelles sans apparaitre particulièrement sous-spécifiés en termes sémantiques. Dans cet article, nous choisirons de privilégier la dimension syntaxique : seront qualifiés de sousspécifiés les noms qui entrent dans la réalisation des structures spécificationnelles, indépendamment de leur caractère plus ou moins abstrait.

10. Ces lexèmes y sont désignés sous le terme de «noms évaluatifs».

11. Une conséquence de l'absence d'occurrence orale est l'impossibilité d'analyser dans le cadre de cet article la prosodie de la construction étudiée.

12. Un second inconvénient bien connu de l'emploi de Google est de laisser le plus souvent dans l'ombre les caractéristiques des locuteurs (origine géographique, profil sociolinguistique...). L'absence de contrôle sur ce que l'on observe est ici maximale.

13. Pour ne prendre qu'un exemple, les suites en je je (ou je j'), que chacun décrirait comme disfluentes, totalisent tout de même près de 11.000 occurrences dans le FWC.

14. Le fait de voir dans ces structures des formes conventionnalisées n'empêche évidemment pas que certaines occurrences particulières puissent résulter d'accidents de performance scripturale liés à l'usage du clavier : tout le problème est qu'il n'est pas possible de distinguer formellement entre ce qui est le produit de la « volonté » des locuteurs / scripteurs et ce qui ne l'est pas ; cette indécision est évidemment de nature à jeter un doute sur la validité de certaines données.

15. Nous avons demandé à 10 locuteurs francophones de lire à voix haute ${ }^{7 \text { phrases }}$ dans lesquelles est c'est était précédé des lexèmes fait, preuve, problème, raison, souci, regret.

16. http://www.festivalregardscroises.com/wp-content/uploads/2016/09/cr3.pdf 
17. Cf. notamment Tuggy 1995, Brenier \& Michaelis 2005, Keizer 2013, O’Neill 2014.

18. Au plan des usages, la structure semble incomparablement plus fréquente en anglais américain parlé qu’en français (Brenier \& Michaelis 2005, 57). Elle paraît en revanche rare en anglais écrit formel : dans son étude portant sur l'analyse systématique d'un important corpus écrit consacré aux "shell nouns ", Schmid (2000, 52) constate l'absence totale d'exemples du type the thing is is that... L'auteur rend compte de l'absence de telles constructions dans son corpus en remarquant qu'elles constituent un phénomène notoirement oral (« notorious phenomena in spoken English »).

19. https://www.escappelle.com/actualites-du-club/c-est-une-grande-satisfaction-de-gagnercontre-le-premier-295843

20. On note que la graphie le soucis n'est pas rare.

21. https://www.agoravox.fr/actualites/societe/article/lait-ble-les-agriculteurs-183743

22. https://droit-finances.commentcamarche.com/forum/affich-3849553-rupture-de-cdi-enperiode-d-essai

23. http://forum-auto.caradisiac.com/pole-technique/mecanique-electronique/sujet193653.htm

24. Une recherche systématique de séquences en [chose + Adj] ou [Adj + chose] (la plus grande chose, la seule chose...) aurait à coup sûr livré un plus grand nombre d'attestations.

25. http://clicimage.eklablog.com/les-ecureuils-sont-prets-pour-affronter-l-hiver-2-a2044224

26. http://www.cours-de-droit.net/la-duree-du-droit-d-auteur-a127390562

27. http://lagourmandisedeviolette-com.over-blog.com/2016/12/toast-au-pain-d-epice-et-foiegras.html

28. https://businessdigital.fr/le-reve-dun-spa-dans-un-palace-avec-les-conseils-de-christelspasdeluxe/

29. http://www.lefigaro.fr/international/2011/03/27/01003-20110327ARTFIG00289-la-situationse-degrade-a-la-centrale-de-fukushima.php

30.

http://www.allocine.fr/communaute/forum/ message_gen_nofil=551385\&cfilm=126276\&refpersonne=\&carticle=\&refserie=\&refmedia=.html 31. http://frontlibertaire.canalblog.com/archives/2012/02/02/23424953.html

32. http://www.mondepluriel.org/le-kit-de-mobilisation-climat/108-le-salon-europeen-de-leducation-rencontre-avec-hubert-reeves-et-presentations-de-projets-par-les-jeunes

33. https://www.commentcamarche.net > Forum > Jeux vidéo

34. https://www.stop-dependance.ch/cgi-bin/alcool/fr/aff_tem2.pl?aff+T1+140

35. Sauf dans cet exemple en la preuve en était c'était que... : Il passa une nouvelle fois sa main sur sa nuque, y avait un risque car si il ne restait pas à sa place alors il se ferait surement viré de tout plateau en même temps Jason savait que Jordan n'était pas n'importe qui la preuve en était c'était qu'il avait parlé à ce réalisateur et qu'il l'avait écouté. [FWC - http://lights-andglitters.forumactif.org/t66-silence-action-take-56-pv-friday-13th]

36. https://travail-vie-pratique.aufeminin.com/forum/probleme-avec-solde-de-tout-comptefd79936

37. https://forum-juridique.net-iris.fr/finances-fiscalite-assurance/212042-tort.html

38. https://www.booking.com/hotel/at/westbahnhof-hostel-wien.fr.html

39. Nous ne poursuivons pas l'objectif de décrire ici le fonctionnement pragmatique ou textuel de ces structures.

40. Ou Sujet-Verbe-Attribut.

41. Même si, comme le relèvent Legallois \& Gréa (2006, 167, note 1), les structures spécificationnelles dont le lexème initial est au pluriel sont peu attestées.

42. Les lexèmes du type fait, truc, chose, qui se caractérisent par leur faible charge lexicale (cf. Blanche-Benveniste, 2010, 10), acceptent très mal ces manipulations (?? qu'est-ce qui est la chose??? Quel est le fait?) et devraient recevoir un autre type d'analyse, que nous ne développerons pas ici. 
43. Ce type de transformation n'est pas recevable avec tous les noms sous-spécifiés. Et même lorsqu'il l'est, Legallois \& Gréa $(2006,163)$ remarquent que les énoncés obtenus sont souvent «lourds ».

44. Cf. Apothéloz \& Roubaud (2015) et Kuyumcuyan (2018) pour un examen des approches en présence.

45. C'est ainsi qu'elle évoque le cas des «valences nominales [qui] se manifestent dans la tournure pseudo-clivée 'le N c'est que P' » (197).

46. Cf. Sabio \& Benzitoun (2013), Roubaud \& Sabio, 2018.

47. Inspirée de Roubaud (2000) et de Blanche-Benveniste (2002-b).

48. Les énoncés en le fait est que, et dans une moindre mesure ceux en chose et truc ont des propriétés particulières et nécessiteraient une analyse spécifique que nous n'aborderons pas ici. Nous reviendrons rapidement aux énoncés en le fait est que dans la dernière partie.

49. Apothéloz $(2008,77)$

50. Remarque de Valli $(1981,197)$.

51. Valli (1981) fait l'hypothèse que l'absence de c' n'est possible que pour une sous-partie des «noms phrastiques» (202-203) : nous ne développerons pas cet aspect ici.

52. Choisis ici car on les retrouve souvent dans les structures non prototypiques en est c'est.

53. La quasi-absence de la forme le fait, c'est que est un indice du caractère fortement figé de cette tournure.

54. L'expression le fait est que fait à nouveau exception: V. Lenepveu $(2009,3)$ confirme que être est toujours au présent.

55. On note d'ailleurs que le clitique en est très fréquent dans les structures spécificationnelles ( la preuve en est que»), ce qui illustre le fait que la rection nominale du nom preuve est d'emblée saturée (sous forme clitique) dès le premier segment de la structure ; si bien qu'on ne voit pas quel statut rectionnel on pourrait donner à la séquence placée à droite de la copule.

56. Nous ne nous prononçons pas ici sur le caractère généralisable des remarques qui viennent d'être formulées. Une étude de détail serait nécessaire.

57. Cette analyse a été suggérée pour les énoncés anglais du type the problem is is that he left early, parfois considérés comme des pseudo-clivées incomplètes, desquelles le pronom initial aurait sauté : cf. what the problem is, is that he left early. L'analyse est proposée dans Massam (1999), et critiquée dans Brenier \& Michaelis (2005).

58. Par ex. Shapiro \& Haley (2002).

59. Dans leur typologie des « amalgames ", Lambrecht \& Ross-Hagebaum $(2006,15)$ décrivent les énoncés anglais en is is comme des phénomènes d'intrusion, qu'ils caractérisent ainsi : “A grammatical morpheme or sequence of morphemes ('ve, is, is that, but also certain kinds of open proposition) is inserted into an otherwise complete and well-formed construction, adding a semantic or pragmatic nuance to the proposition".

60. A nouveau, le tableau ne s'applique pas à le fait est que / le fait est c'est que (voir remarque plus loin).

61. En voici un exemple oral : «pour ceux qui sont plus jeunes qui naissent qui sont les enfants de ces gens-là euh ils ont peut-être le désir de choisir et c'est là que le problème se pose c'est que effectivement on peut leur demander on peut on pourrait dire que: le droit du sol serait euh: accordé (...) (oral, cité par Teston-Bonnard 2018, 80)

62. Cf. Lenepveu $(2009,12)$.

63. C. Blanche-Benveniste (1990) y voyait un fait de « grammaire seconde ». 


\section{RÉSUMÉS}

Les énoncés spécificationnels à nom sous-spécifié du français peuvent se réaliser sous une forme non prototypique qui réalise deux fois le verbe être : le problème est c'est que... Les données indiquent que ces constructions sont peu fréquentes; elles semblent toutefois suffisamment bien attestées - notamment avec certains lexèmes tels que preuve, fait, problème - pour qu'on puisse y voir une structure grammaticale et non la simple manifestation d'«accidents » de performance. Au plan grammatical, nous avons proposé de les analyser comme des structures qui superposent deux emplois distincts du verbe être : d'abord, un emploi copulatif dans lequel la forme verbale a la particularité de pouvoir varier en temps (est, était, étant...); en second lieu, la forme c'est, à laquelle nous accordons la valeur d'un auxiliaire de dispositif, comme on en trouverait dans les énoncés clivés ou pseudo-clivés.

In French, specificational utterances containing an under-specified noun are likely to take a nonprototypical form in which the verb être [be] is realized twice: le problème est c'est que... [lit. the problem is it is that...]. Corpora indicate that although infrequent, such utterances are sufficiently well attested - especially with certain nouns such as preuve, fait, problème [proof, fact, issue] - to allow us to consider them as fully grammatical constructions rather than mere disfluencies. In terms of syntactic structure, we suggest describing them as constructions that amalgamate two distinct values of the verb être [be]: firstly, the value of a copula which licenses some temporal and apectual variation (est, était, étant [is, was, being]); secondly, the invariable form c'est [it is] which is described as a kind of auxiliary verb one would find in clefts or pseudo-clefts constructions.

\section{INDEX}

Mots-clés : constructions spécificationnelles, énoncés pseudo-clivés, verbe copule, noms sousspécifiés

Keywords : specificational constructions, pseudo-clefts, copula, under-specified nouns

\section{AUTEUR}

\section{FRÉDÉRIC SABIO}

Aix-Marseille Université \& CNRS Laboratoire Parole et Langage 